\title{
Gut Microbiota Development: Influence of Diet from Infancy to Toddlerhood
}

\section{Martin Frederik Laursen}

National Food Institute, Technical University of Denmark, Kongens Lyngby, Denmark

\section{Key Messages}

- The gut microbiota develops drastically during the first year of life and is influenced by a range of external factors, with diet being a major player.

- Breastfeeding versus formula feeding strongly affects gut microbiota composition and metabolism during early infancy. Breastfeeding promotes the dominance of specific human milk oligosaccharide-degrading Bifidobacterium species in the gut, which may contribute to protection against infectious and immune-related diseases. Formula feeding results in a more diverse gut microbiota with a higher prevalence of opportunistic pathogenic bacterial taxa and proteolytic metabolism in the gut.

- Complementary feeding and the increasing dietary fiber and protein intake induces a shift from the milk-adapted gut microbiota and metabolism toward one with vastly increased diversity and proportions of the fiber-degrading bacterial families Lachnospiraceae, Ruminococcaceae, and Bacteroidaceae, and their major metabolic end products short chain fatty acids. Inadequate maturation of the gut microbiota during complementary feeding is associated with poor growth and development in early life.

\section{Keywords}

Gut microbiota · Diet · Breastfeeding · Complementary feeding

\section{Abstract}

Early life is a critical period as our gut microbiota establishes here and may impact both current and future health. Thus, it is of importance to understand how different factors govern the complex microbial colonization patterns in this period. The gut microbiota changes substantially during infancy and toddlerhood in terms of both taxonomic composition and diversity. This developmental trajectory differs by a variety of factors, including term of birth, mode of birth, intake of antibiotics, presence of furred pets, siblings and family members, host genetics, local environment, geographical location, and maternal and infant/toddler diet. The type of milk feeding and complementary feeding is particularly important in early and late infancy/toddlerhood, respectively. Breastfeeding, due to the supply of human milk oligosaccharide into the gut, promotes the growth of specific human milk oligosaccharide (HMO)-utilizing Bifidobacterium species that dominate the ecosystem as long as the infant is primarily breastfed. These species perform saccharolytic fermentation in the gut and produce metabolites with physiological effects that may contribute to protection against infectious and immune-related diseases. Formula feeding, due to its lack of HMOs and higher protein content, give rise to a more diverse gut microbiota that contains more opportunistic pathogens and results in a karger@karger.com

www.karger.com/anm (c) 2021 S. Karger AG, Basel

$$
\text { 嵉 }
$$

Correspondence to:

Martin Frederik Laursen, mfrla @ food.dtu.dk 
more proteolytic metabolism in the gut. Complementary feeding, due to the introduction of dietary fibers and new protein sources, induces a shift in the gut microbiota and metabolism away from the milk-adapted and toward a more mature and diverse adult-like community with increased abundances of short chain fatty acid-producing bacterial taxa. While the physiological implication of these complementary diet-induced changes remains to be established, a few recent studies indicate that an inadequately matured gut microbiota may be causally related to poor growth and development. Further studies are required to expand our knowledge on interactions between diet, gut microbiota, and health in the early life setting.

(C) 2021 S. Karger AG, Basel

\section{Key Insight}

The healthy fetus is devoid of microbial organisms, but upon birth our gastrointestinal (GI) tract becomes colonized with a multitude of microbes, gradually developing into a complex microbial community during the first year of life. This community, termed the gut microbiota, interacts with our gut epithelium, immune, and nervous systems and influences our metabolism. During the last decades, the gut microbiota has been linked with a range of gut inflammatory, infectious, allergic, autoimmune, neurological, and metabolic diseases. Thus, understanding the processes that govern initial colonization and establishment of microbes in our Gl tract is of great importance. A key factor influencing the gut microbiota development in early life is diet. This article review our current knowledge of how the type of milk feeding and complementary feeding affects the microbial ecosystem in the gut and how this may impact current and future health.

\section{Current Knowledge}

Type of milk-feeding and complementary feeding dictates the microbial succession throughout infancy and into early toddlerhood. Breastfeeding keeps the gut microbiota in a state of low diversity dominated by human milk oligosaccharide-utilizing Bifidobacterium species that produce various metabolites characteristic of saccharolytic fermentation. Formula feeding allows for a somewhat more diverse gut microbiota to colonize and is characterized by higher prevalence of potential pathogenic taxa such as Clostridium and Enterobacteriaceae and a higher degree of proteolytic fermentation. The gradual cessation of milk-based feeding and progression in complementary feeding, characterized by increased consumption of dietary fibers and proteins, results in a further diversification and maturation of the microbial community in the gut with increased abundance of the bacterial families Lachnospiraceae, Ruminococcaceae, and Bacteroidaceae, as well as expansion of bacterial products from saccharolytic and proteolytic metabolism.

\section{Practical Implications}

The breastfeeding promoted Bifidobacterium species produce metabolites that lower intestinal $\mathrm{pH}$ and thus creates an environment hostile to pathogenic bacteria, they exert antiinflammatory properties, protect the gut epithelium and regulate the immune system. These properties may result in reduced the risk of infectious and immune-related diseases for breastfed infants harboring these species. Formula fed infant are more frequently colonized with opportunistic pathogenic and toxin-carrying bacterial species and exhibit a mostly proteolytic metabolism in the gut, which may influence the risk of infectious, but also metabolic and immune-related diseases. Lack of diet-promoted maturation of the gut microbiota during complementary feeding is associated with poor growth and neuro, bone and immune development, although the evidence base for this is extremely limited at present. Further studies disentangling the complex interactions between infant diet, gut microbiota and health are warranted in order to approach clinical implication.

\section{Recommended Reading}

Laursen MF, Bahl MI, Licht TR. Settlers of our inner surface: factors shaping the gut microbiota from birth to toddlerhood. FEMS Microbiol Rev. 2021;1:1-14 [1].

\section{Gut Microbiota Development in Early Life}

Despite recent controversies, the general consensus is that the healthy fetus is devoid of microbial organisms [2]. During and following birth the neonatal external body surfaces and gastrointestinal (Gl) tract rapidly becomes colonized with a multitude of microorganisms, reaching $>10^{8}$ microorganisms per gram feces in a matter of hours to days [3] and over weeks to months increase up to $10^{11}-10^{12}$ microorganisms per gram feces $[4,5]$, comparable to the microbial densities found in the adult gut $[6,7]$. The infant gut microbiota undergoes drastic changes during the first years of life in terms of taxonomic 


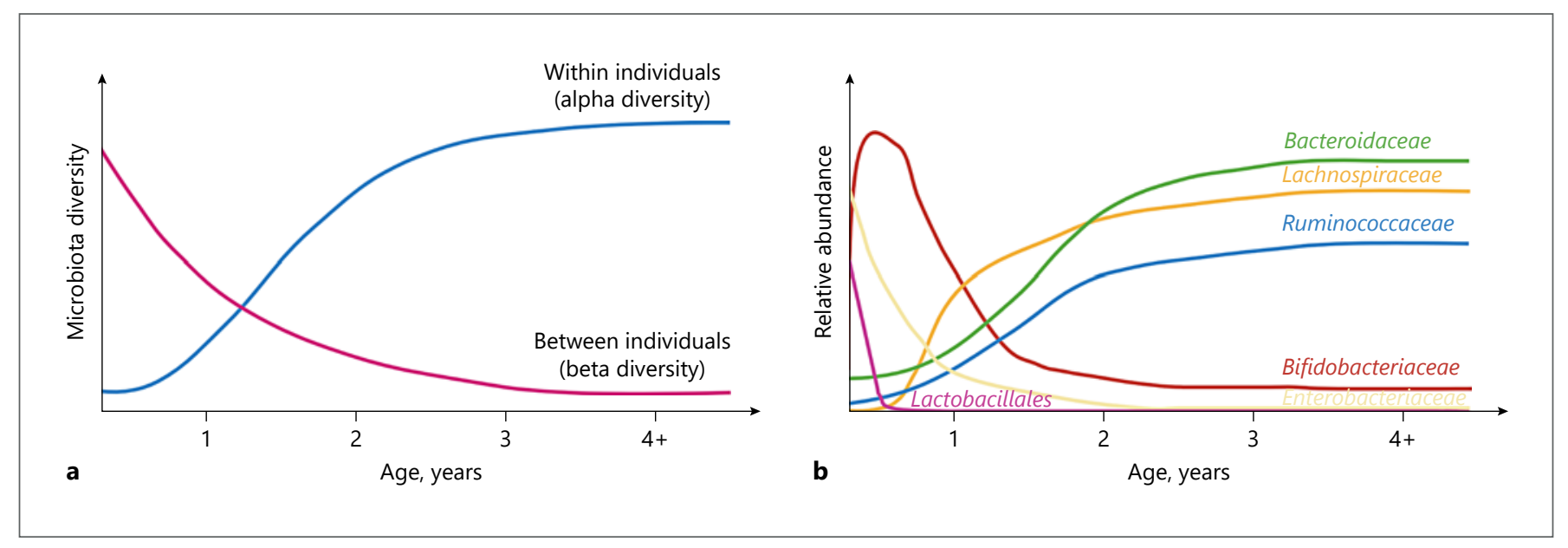

Fig. 1. Development of the gut microbiota in early life. a Development of gut microbiota diversity with age [97]. b Development of the average gut microbiota composition with age, showing the relative abundance of the major microbial families/orders [11, 13]. composition and diversity (Fig. 1). As a function of age, the infant becomes exposed to and colonized by a range of new microbes, increasing diversity within the individual (termed alpha diversity). However, differences in gut microbiota composition between individuals (termed beta diversity), is initially high, resulting from individual exposures to various different environmental sources of microbes, but these differences gradually decrease as selective forces, such as diet, converge microbiota compositions (Fig. 1a). Thus, the individual infant continuously acquires new microbial taxa with age, but when comparing across infants the gut microbiota gradually becomes taxonomically more similar. Aerotolerant and facultative anaerobes, such as lactic acid bacteria (Lactobacillales genera, such as Streptococcus, Enterococcus and Lactobacillus) and Enterobacteriaceae are among the major initial colonizers of the neonatal gut, but their proportions rapidly decrease within days to weeks and the gut microbiota in breastfed (BF) infants becomes dominated by anaerobic breast milk-promoted Bifidobacteriaceae. As the gut microbiota develops during infancy and early childhood, abundance of other anaerobic bacterial families, such as Bacteroidaceae, Lachnospiraceae, and Ruminococcaceae increase (Fig. 1b). In early life, the gut microbiota is unstable and less resilient to perturbation than the adult gut microbiota [8], but the ecosystem develops in alpha diversity until around 3-5 years of age, where a stable adult-like microbiota has established [9]. However, before this state is reached, the gut microbiota is more susceptible to modulation by external factors. Factors influencing the infant gut microbiota development include (as reviewed in [10]); term of birth, mode of birth, intake of antibiotics, presence of furred pets, host genetics, siblings and family members, geographical location/population, growing up environment (i.e., urban vs. rural), maternal diet (through breast milk), and infant diet (Fig. 2). While all of these individually have shown impact, infant diet has been identified as a major contributor to gut microbiota development in early life [11, 12]. In this regard, early infancy diet, such as formula versus breast milk has been relatively well studied, whereas much less is known about the effects complementary feeding on infant gut microbiota composition [13]. Given the dominating influence of breastfeeding on gut microbial composition as well as its protective effects against infectious and some metabolic diseases [14], and the involvement of gut bacteria in regulation of host immune system and metabolism [15], it is of great importance to obtain a deep understanding of the potential microbe-mediated host effects of feeding mode in early infancy. Also, the complementary feeding period (6-24 months of life) coincides with a critical period in gut microbiota development, transitioning away from the influence of milk-based diet. As early childhood development of a mature and diverse adult-like gut microbiota, which is stable and resilient toward perturbation, appears to be an important asset of health [16], assessing how dietary changes in early life affect gut microbiota trajectory is of great value.

\section{Early Infancy Feeding and Gut Microbiota}

Breast milk is the recommended first nutrition for the infant, providing all necessary nutrients to support growth and development, as well as passive immunity to protect against infectious diseases during infancy [17]. After lactose and lipids, 


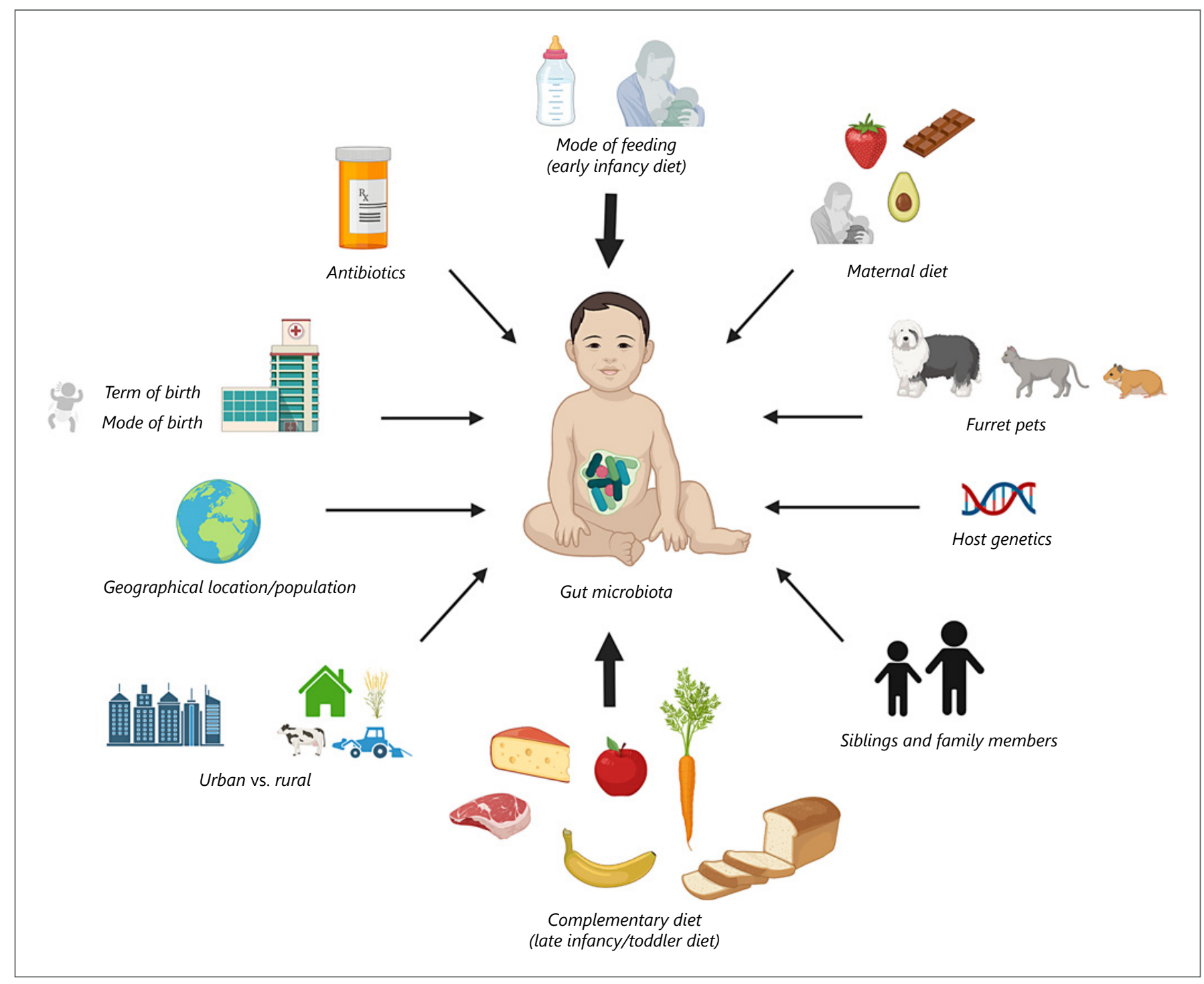

Fig. 2. Factors influencing the gut microbiota in early life. Gut microbiota vary by term of birth, mode of birth, oral antibiotics, mode of feeding, maternal diet, presence of furred pets, host genetics, presence of siblings and family members, complementary diet, and local

human milk oligosaccharides (HMOs) represent the third most abundant component of breast milk, reaching 20-25 $\mathrm{g} / \mathrm{L}$ in colostrum and ranging between 5 and $15 \mathrm{~g} / \mathrm{L}$ in mature milk [18]. HMOs are short saccharides composed of 5 monomeric building blocks (Glucose, Galactose, N-acetylglycosamine, Fucose, and Sialic acid). From these 5 building blocks, >200 different HMOs structures have been identified [19]. Whereas lactose, protein, and lipid serve as important macronutrients for the BF infant, HMOs are virtually ingestible by the infant's own Gl saccharolytic enzymes. Therefore, HMOs pass through the upper Gl tract and reach the colon largely intact. environment, such as urban versus rural and geographical location/ population [10]. Mode of milk feeding and complementary diet has been identified as the strongest determinants of infant gut microbiota composition $[11,12,89]$ as indicated by the arrow sizes.

Throughout the Gl tract, they can exert direct biological functions, such as antiadhesion of pathogens and modulation of epithelial cell responses [18], but in the colon they also serve as metabolic substrates for gut bifidobacteria. Some of these bifidobacteria harbor an arsenal of membrane transporters and saccharolytic enzymes which cleave and internalize HMOs [20] to implement the monomers into the central catabolic pathways, leading to the main end products lactate, acetate, formate, and 1,2-propandiol [21-24] (Fig. 3a). Although other bacteria (e.g., Bacteroides, Lactobacillus, and recently the Roseburia/Eubacterium group) exhibit some de- 


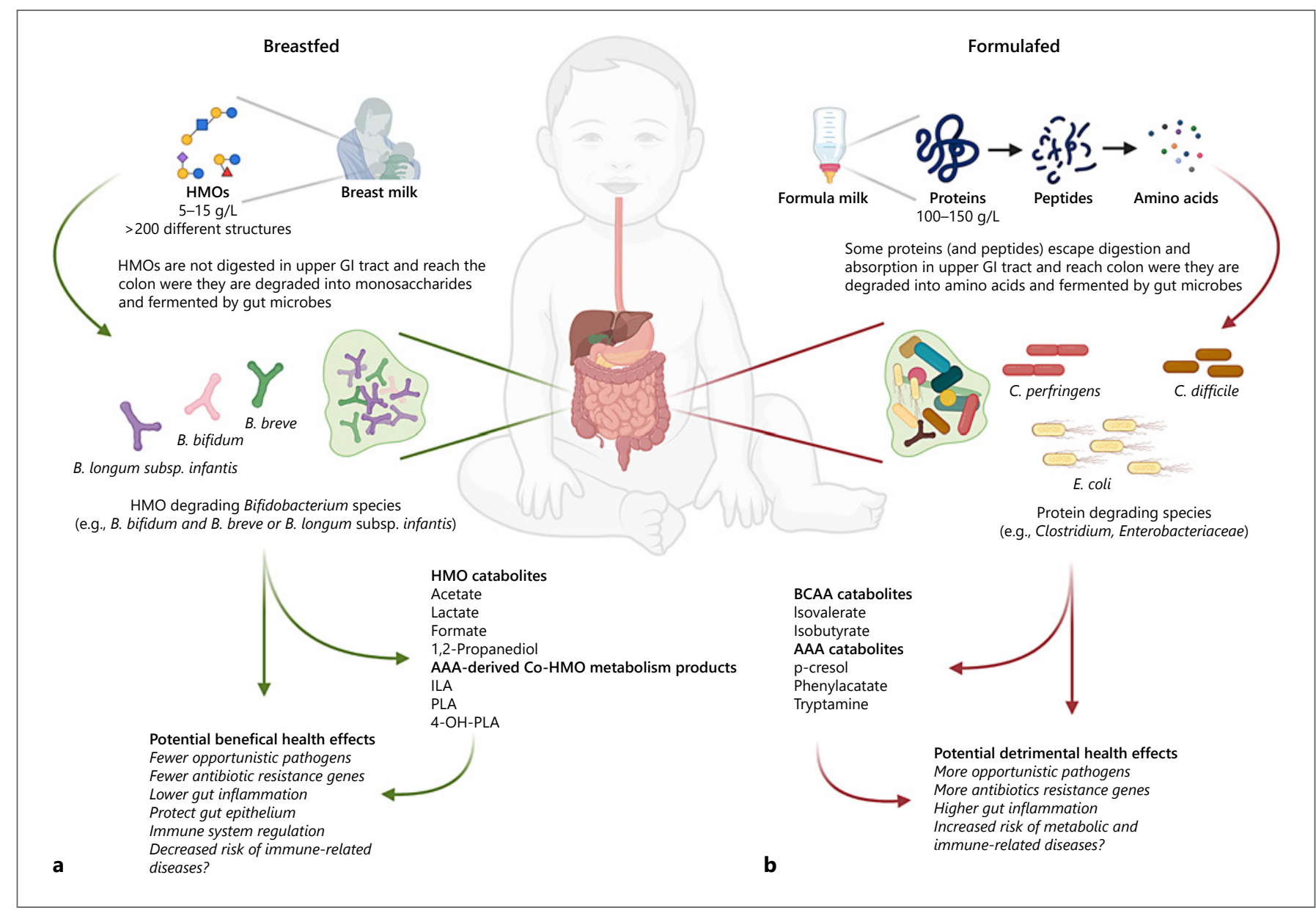

Fig. 3. Mode of feeding influences early infancy microbiota and saccharolytic versus proteolytic fermentation in the gut with potential implications for health. a Breast milk contains HMOs, which upon ingestion by the infant pass undigested through the upper Gl tract until they reach the colon. Here, they are digested by specific HMOdegrading Bifidobacterium species into various metabolites with potential beneficial health effects. b Formula milk contain excess proteins, some of which, upon ingestion, is incompletely digested and absorbed in the upper Gl tract and reaches the colon. Partly digest-

gree of HMO utilization [25-27], the extensive arsenal of enzymes and transporters is restricted to specific species within the Bifidobacterium genus. Whereas some species, such as $B$. bifidum, employ extracellular saccharolytic enzymes to degrade HMOs and internalize mono- and disaccharides, other species such and $B$. breve and especially $B$. longum subsp. infantis transport the intact $\mathrm{HMO}$ structures and degrades them inside the cell [20]. The ability of these species to efficiently utilize HMOs makes them dominant members of the gut of BF infants $[11,28,29]$. Importantly, other Bifidobacterium species commonly found in the adult gut (e.g., B. ado- ed protein, peptides, and individual amino acids are metabolized by gut microbes, including opportunistic pathogenic bacteria, such as Clostridium and Enterobacteriaceae species, yielding various amino acid catabolites, some of which have potential detrimental health effects. HMO, human milk oligosaccharide; AAA, aromatic amino acid; $B C A A$, branched chain amino acid; Gl tract, gastrointestinal tract; ILA, indolelactate; PLA, phenyllactate; 4-OH-PLA, 4-hydroxypheyllactate.

lescentis, B. catenulatum, and B. angulatum) cannot utilize HMOs and therefore are less abundant and/or infrequent members of the gut microbiota of BF infants [20]. Thus, due to the HMO content, breast milk represents a strong selective factor for shaping the early infancy microbiota, dominated by specific Bifidobacterium species (Fig. 3a). Through HMO catabolism, these Bifidobacterium species produce metabolites with potential host-health effects. Acetate and lactate are 2 main end products of bifidobacterial $\mathrm{HMO}$ metabolism and are responsible for the low $\mathrm{pH}$ found in feces from $\mathrm{BF}$ infants $[24,30,31]$, which is likely to suppress the growth of oppor- 
tunistic pathogenic species within Clostridiaceae, Enterobacteriaceae, and Staphylococcaceae [30, 32]. Further, Bifidobacterium-produced acetate was found to inhibit enteropathogenic $E$. coli infection in a mouse model, by maintaining the epithelial barrier function upon insult [33]. HMO-utilizing Bifidobacterium species are also main producers of a limited set of the aromatic amino acid catabolites, namely indolelactate (ILA), phenyllactate, and 4-hydroxypheyllactate (4-OHPLA), in the gut of BF infants [29]. These metabolites are produced when the bacteria grow on HMOs (and have access to the aromatic amino acids tryptophan, tyrosine, and phenylalanine, also contained in breast milk), and interact with various receptors expressed in immune cells, exhibit immunomodulatory potential and protect the gut epithelium [29, 3436]. This may contribute to the anti-inflammatory status observed in the Gl tract of BF infants with Bifidobacteriumrich communities [37]. However, despite being BF, some individuals (e.g., infants born preterm, by $\mathrm{C}$-section or in population with high consumption of antibiotics which disrupts vertical transmission from the maternal gut) lack Bifidobacterium species, such as B. longum, B. breve, and B. bifidum [38, 39]. Lack of Bifidobacterium is associated with immune dysregulation [40] and development of asthma [41] and autoimmune diseases [42], but early life intervention with a HMO-degrading $B$. longum subsp. infantis strain in BF infants modulates immune responses away from the allergy and autoimmunity associated immune-phenotypes [40]. In both full term [31] and preterm $[43,44]$ BF infants lacking bifidobacteria, oral administration of $\mathrm{HMO}$-utilizing Bifidobacterium spp. were found to decrease the abundance of opportunistic pathogens, antibiotic resistance genes, and reduce intestinal inflammation, demonstrating the importance of this group of bacteria for infant health (Fig. 3a).

On the contrary, exclusively formula-fed (FF) infants harbor a more diverse microbiota with lower abundances of $\mathrm{HMO}$-utilizing Bifidobacterium species, often with increased abundances of Clostridium species (C. difficile and C. perfringens) and Enterobacteriaceae species (e.g., E. coli) [45-47] (Fig. 3b). The lack of HMOs and higher protein content in formula is likely to explain these observations. Although many infant formula products are supplemented with fructo-oligosaccharides and/or galacto-oligosaccharides, these are not as selective since they can be utilized by most Bifidobacterium species (including the adult-associated $B$. adolescentis and $B$. catenulatum) $[48,49]$ and additional can stimulate growth of various Lactobacillus, Streptococcus, and Bacteroides species $[50,51]$. Studies of the fecal metabolome in exclusively FF (even when formula is containing galacto-oligosaccharides) versus exclusively BF infants, suggest that proteolytic rather than saccharolytic metabolism dominates in the FF gut $[52,53]$. Whereas remnants of $\mathrm{HMO}$ metabolism (e.g., fucose), their direct catabolic end products (e.g., lactate and 1,2 propanediol) and aromatic amino acid derived coHMO metabolism products [29, 35] (e.g., indolelactate and 4-hydroxypheyllactate) dominate in BF infants, various different protein fermentation products such as isovalerate, isobutyrate, phenylacetate, $\mathrm{p}$-cresol and tryptamine dominate in FF infants [52, 53] (Fig. 3). Some of these metabolites (e.g., $\mathrm{p}$-cresol and phenylacetate) are converted in the liver to metabolites with detrimental effects, such as p-cresol-sulfate, which is a uremic toxin [54] and phenylacetateglutamine which may contribute to development cardiovascular diseases [55]. Phenylacetate has also been shown to promote accumulation of fat in the liver [56]. Further, bacterially produced tryptamine in FF infant gut may shift tryptophan metabolism away from serotonin and influence immune system development [57]. Biogenic amines produced by bacterial amino acid metabolism (such as tryptamine and histamine) can cause Gl symptoms and allergic reactions [58, 59]. In addition $C$. perfringens and $C$. difficile that often colonize the gut of FF infants are potential toxin-carrying opportunistic pathogens $[60,61]$ and the gut microbiota of FF infants has a higher abundance of antimicrobial resistance genes [62, 63]. Last, FF infant have higher levels of inflammation markers than BF infants [64].

In summary, breastfeeding, due to the presence of HMOs, promotes growth of beneficial bifidobacteria in the infant gut, which produces metabolites that may contribute to prevention of Gl infections and support immune system development. By contrast, formula feeding leads to a gut microbiota with higher abundances of opportunistic pathogens and a mostly proteolytic gut metabolism, leading to potentially detrimental health effects (Fig. 3). However, it has recently become technically and legally possible to add synthetically produced HMOs (e.g., 2'FL) to infant formula in order to stimulate a BF-like gut microbiota and metabolism [65]. A recent double-blinded randomized controlled trial investigated how addition of 2 HMOs, namely $2^{\prime} F L$ and LNnT, to infant formula influence the gut microbiota, and compared HMO-supplemented and unsupplemented formula groups with a reference group of BF infants [66]. The addition of HMOs to the formula resulted in a microbiota configuration that was more similar to that of the BF infants. Infants receiving $\mathrm{HMO}$-supplemented formula, similar to the BF infants, had higher relative abundances of Bifidobacterium and lower relative abundances of Enterobacteriaceae and Peptostreptococcaceae (the bacterial family that includes $C$. difficile) compared to infants receiving unsupplemented formula. Although promising, this study is the first of its kind in investigating the influence on the gut microbiota and confirmatory studies are re- 
quired. Nonetheless, this strategy may constitute an avenue to improve the gut microbiota in infants that for some reason cannot be BF.

\section{Complementary Feeding and Gut Microbiota}

At some point during infancy milk-based feeding is no longer adequate to cover the nutritional requirements of the infant. Therefore, supplementation with additional foods, alongside milk-feeding, is needed. The period of transition from exclusive milk-feeding toward eating family foods is termed complementary feeding and usually covers age 6-24 months [67, 68]. It is recommended by $\mathrm{WHO}$ to start complementary feeding around 6 months of age [67]; however, in some countries/populations is it not uncommon to introduce other foods as early as 2-3 months of age [69]. Early complementary feeding (before 3 months of age) has been linked to increased risk of $\mathrm{Gl}$ and respiratory infections, obesity, and allergies, but this may rather be attributed to shorter duration of breastfeeding [70]. However, late introduction to complementary feeding can also be problematic as it may result in feeding problems, inadequate nutrition and growth [70], and failure to induce oral tolerance [71]. Recent evidence suggests that the infant gut microbiota development may be causally linked to healthy growth $[72,73]$ and protection against food allergies [74, 75].

Despite extensive progression, during the last decade, in our understanding of gut microbial succession in early life, and our continuously expanding knowledge about microbial capacity to consume various dietary compounds, we know surprisingly little about causal effects of diet on gut microbiota during complementary feeding. As outlined above, the complementary feeding period coincides with a phase of drastic changes in the gut microbiota (Fig. 1), including a rapid decline in $\mathrm{HMO}$-degrading Bifidobacterium species. The prominent increase in alpha diversity and appearance/bloom in Bacteroidaceae, Lachnospiraceae, and Ruminococcaceae species, mirror the increased complexity of the diet with the introduction of fibers from various fruits, vegetables, cereals/porridge, and breads as well as new protein sources that is, in the form of meats, dairy products, and legumes/lentils. As dietary fibers and secondly (incompletely digested) proteins/peptides are the main sources of energy for gut microbes [76] these macronutrients would be expected to have most impact on the microbial composition. Carbohydrates (dietary fibers) are preferred energy-sources for microbes, but in shortage of these a higher degree of proteolytic fermentation occurs in the gut (as illustrated in the example of metabolism in the gut of FF infants mentioned above). This balance not only depends on diet but will also vary with passage of luminal content through the colon, as dietary fibers will gradually become depleted [76]. The main catabolic end products of dietary fiber metabolism are the short chain fatty acids (SCFAs) acetate, butyrate, and propionate [77]. Whereas the former (together with lactate, formate, and succinate) is produced in high quantities in early infancy (e.g., by Bifidobacterium, Lactobacillus, and Enterobacteriaceae spp.), butyrate and propionate concentrations are initially very low but increase with infant age [24]. In addition, products of protein fermentation, such as branched chain fatty acids (BCFAs) are almost undetectable during breastfeeding, but follow a similar pattern of increase with age [24]. These changes in SCFAs and BCFAs are coinciding with the introduction of solid foods and cessation of breastfeeding [24]. Consistent with the typical gut microbiota developmental pattern, some key Lachnospiraceae (Anaerostipes, Roseburia, and Eubacterium) and Ruminococcaceae (Faecalibacterium, Gemminger, and Subdoligranulum) species are butyrate producers $[24,78]$, whereas Bacteroides are common propionate producers [79]. These species harbor an extensive catalog of enzymes (glycosyl hydrolases) for degradation of dietary fibers [80] into these SCFAs [79]. In addition, some Bacteroides and Clostridium species may utilize various amino acids from dietary proteins to form BCFAs [81]. Thus, complementary feeding is very likely to causally affect microbiota composition and metabolism. As SCFAs have a range of physiological effects, including influence on intestinal barrier function, host metabolism, immune system, and nervous system [82], these are plausible mediators of microbiota-host interactions during complementary feeding affecting host health (Fig. 4). Much less is known about the physiological effects of BCFAs [83].

Longitudinal studies, designed with multiple samplings around the period of first introduction to solid foods, have demonstrated increased alpha diversity and abundance of Lachnospiraceae genera (such as Blautia) after introduction of solid foods $[84,85]$. Supporting this, earlier introduction to complementary foods is associated with higher microbial alpha diversity throughout infancy, including higher abundance of the butyrate producing Lachnospiraceae genera Roseburia [86]. In a randomized controlled trial [87] the authors compared the gut microbiota in infants that were weaned traditionally (with spoon feeding) to infants weaned with a babyled approach (no spoon feeding, but only complementary "finger foods" consumed), with complementary diet assessed by 3 -day dietary records. The baby-led weaned infants were introduced to solid foods roughly 3 weeks later $(6$ months of age) and consumed significantly less fruits and vegetables and total dietary fiber at 7 months of age, which was associated with a reduced alpha diversity and lower abundance of specific Lachnospiraceae (Roseburia facies and Eubacterium rec- 
Fig. 4. Complementary feeding increases gut microbial diversity and production of BCFAs and SCFAs with potential implication for growth, neuro, bone, and immune development. As complementary feeding progresses, the milk-based component is gradually replaced by other foods such as meats and dairy, fruits and vegetables, and bread and cereals, which are directly and possibly indirectly (through the gut microbiota) impacting growth and development. These foods contain protein and dietary fibers that modulate the infant gut microbiota, increasing alpha diversity and the abundance of key bacterial families that produce SCFAs (note that acetate is also produced in high amount during early infancy) and BCFAs during complementary feeding. This "natural" development of the gut microbiota and its metabolites is associated with healthy growth, neuro, bone development, and appropriate immune system regulation. BCFAs, branched chain fatty acids; SCFAs, short chain fatty acids.

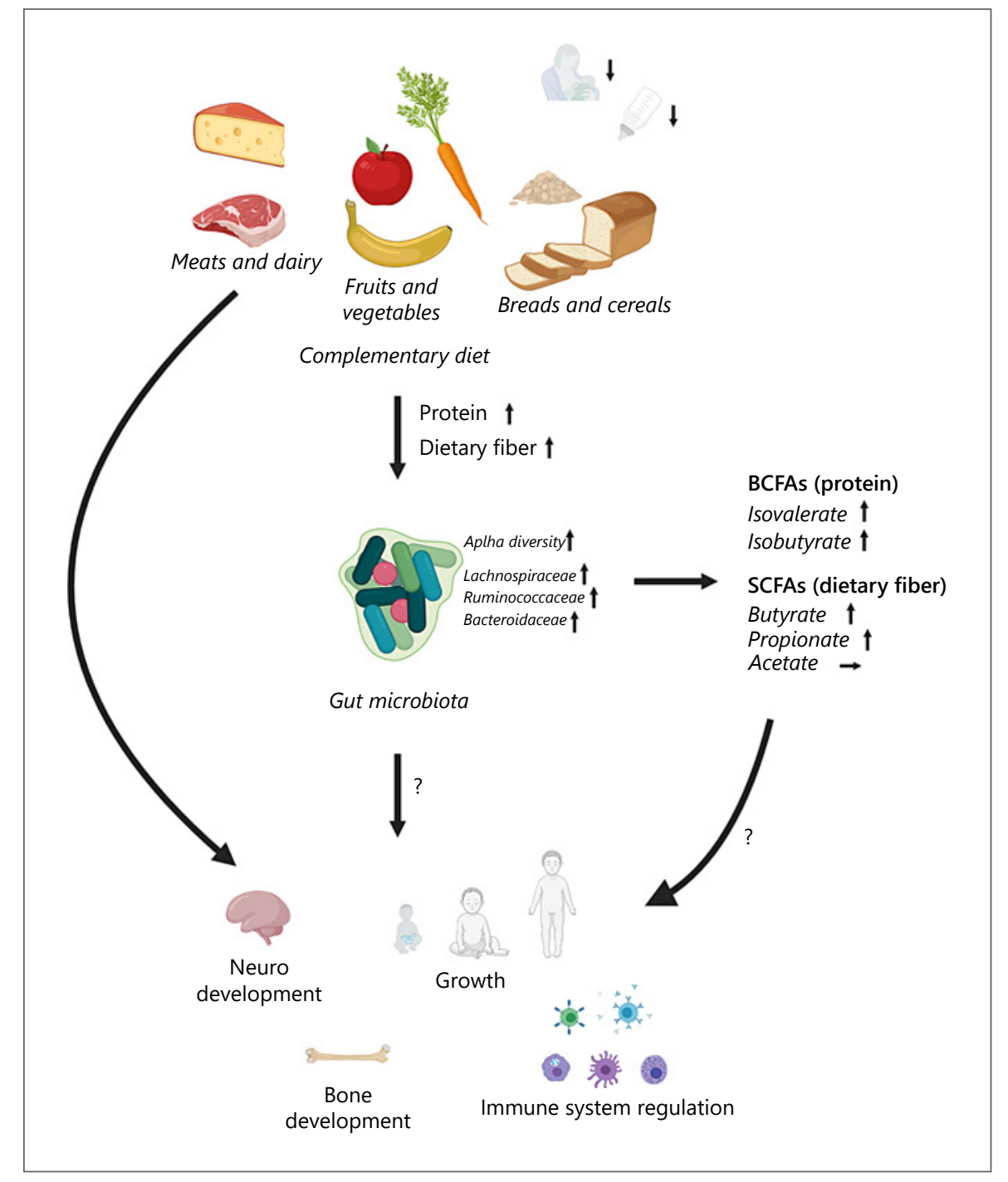

tale) and Ruminococcaceae (Faecalibacterium prausnitzii) species during complementary feeding [87]. In addition, independent of the feeding groups, consumption of breads and cereals, as well as meat products at 7 months of age were positively associated with alpha diversity at 12 months of age [87].

A study including 9 months old Danish infants assessed the complementary feeding diet by 7 -days dietary records and a used multivariate statistical analysis to generate the dietary pattern "family foods" [88, 89], describing the infant's progression in complementary feeding (from milk-based toward family foods). Progression in complementary feeding, characterized by higher dietary fiber and protein intake, correlated significantly with gut microbial alpha diversity [89]. Specifically, consumption of rye bread and cheese and meat prod- ucts were main food groups driving these associations [89], suggesting that these complementary foods are contributing to the diversification of the infant gut microbiota, at least in this cohort. Importantly, these associations were not solely mediated by cessation of breastfeeding, as they were found in both partially BF and partially FF infants [13]. Progression in complementary feeding also correlated positively to the abundance of several Lachnospiraceae and Ruminococcaceae spp., but negatively to Bifidobacterium [13], thus marking the transition of the breast milk promoted Bifidobacteriumrich gut community toward the fiber and protein promoted (more diverse) gut microbial community, characterized by butyrate, propionate, and BCFA-producing bacteria (Fig. 4).

Indeed, interventions with meat as a main complementary food compared to dairy [90] or cereals [91] has revealed 
significantly increased abundance of butyrate-producing Lachnospiraceae genera. A recent 7-week intervention study comparing a refined grain cereal product to a whole grain cereal product as first complementary food, demonstrated a significant increase in Bacteroides and Lachnoclostridium (Lachnospiraceae family) and a decrease in Escherichia (Enterobacteriaceae family) over time only in the whole grain cereal group. However, other intervention with specific complementary foods such as legumes versus corn-soy flour [92] or meat versus cereals [93] show limited differential impact on the gut microbiota, possibly due to these infants still receiving a high proportion of breast milk in their diet or other study participant characteristics. Ultimately, more randomized controlled trials are needed to establish direct causal proof of links between specific complementary foods and specific microbial taxa. Nonetheless, as outlined below, a few recent studies indicate that specific Lachnospiraceae species affected by complementary foods may influence growth and development and protect against food allergies (Fig. 4).

Stunted undernourished Bangladeshi infants/toddlers have an immature gut microbiota compared to same age healthy well-nourished counterparts [94], likely as a result of inadequate and/or delayed complementary feeding. Transplantation of the gut microbiota, using fecal samples from stunted, malnourished versus healthy infants, into germ-free mice (devoid of microbes) has revealed differential growth patterns, recapitulating the growth phenotypes observed in these infants [73]. Oral introduction of some of the Lachnospiraceae species (such as Ruminococcus gnavus and Clostridium symbiosum) that appear during complementary feeding in healthy infants was shown to promote growth and bone development in mice previously transplanted with the malnourished gut microbiota [73]. Furthermore, complementary food intervention in stunted malnourished Bangladeshi toddlers rationally designed to repair the immature gut microbiota suggest that a combination of banana, chickpea, soy, and peanut matures the gut microbiota (increase abundance of some Lachnospiraceae and Ruminococcaceae species) and influence blood markers of healthy growth, bone, immune, and neurodevelopment $[72,95]$. However, despite having a similar energy, macronutrient and fiber content, other rationally designed microbiota-directed complementary food combinations were not effective, illustrating the need for a deeper understanding of the effects of specific food combinations.

Recent studies have demonstrated that blood concentrations of food specific immunoglobulin E (IgE), an important mediator in food allergy, is elevated in germ-free mice compared to conventional (colonized) counterparts after weaning onto an antigen-containing diet [75]. In addition, colonization of previously germ-free mice with gut commensal microbes inhibit the increase in food-specific $\lg E$ and disruption of the gut microbiota by oral administration of antibiotics to conventional mice increases food-specific IgE concentrations in blood [75]. Thus, gut microbes play a causal role in regulation of IgE levels during the food antigen exposure and may affect the risk of developing food allergy. Another study performed gut microbiota transplantation from healthy or cow's milk allergic 6-month old infants into germ-free mice and challenged them with the cow's milk allergen $\beta$-lactoglobulin [74]. In contrast to the mice colonized with the cow's milk allergic infant gut microbiota, mice colonized with the healthy infant gut microbiota were protected against allergic responses. In the healthy infant gut microbiota, Anaerostipes coccae, a Lachnospiraceae species that increases during complementary feeding and associates with consumption of vegetables and fruits [96], was identified as a bacterial taxon that mediated this protection. By comparing mice colonized with cow's milk allergic microbiota to mice mono-colonized with $A$. caccae it was demonstrated that this species protected the mice against allergic food response [74]. While results from these studies are encouraging, it must be emphasized that our current knowledge on the effects of different complementary foods on gut microbiota and thereof potential health impact is still extremely limited.

\section{Future Directions}

Undoubtedly, diet plays a major role for the succession of the infant gut microbiota, yet we still have a very incomplete understanding of the details. There is a need for well-designed and well-powered interventions in various different populations in order to assess the impact of formula supplemented with different HMOs on gut microbiota and metabolome in early infancy, including relevant measures of host impact, such as prevalence of infectious and immune-related diseases, during and after intervention. There is a lack of studies with detailed dietary recordings coupled to measurements of the gut microbiota at a finer resolution scale. In example, to improve our knowledge on dietary factors shaping the infant gut microbiota, we need prospective cohort studies with detailed information about infant diet, obtained from multiple consecutive days of dietary recordings throughout periods of complementary feeding, combined with dense fecal sampling and state of the art microbiome (obtaining species and strain level resolution) and metabolome profiling, as well as deep genomic and phenotypic characterization of fecal microbial isolates to decipher molecular mechanisms. These studies 
should be accompanied by intervention studies, in relevant study populations, designed to introduce specific complementary foods using frequent longitudinal sampling and the state-of-the-art methods of microbiome and metabolome profiling. Hopefully, this will lead to identification of key dietmicrobe-metabolite interactions that beneficially impact aspects of host physiology, such as metabolism and immunology. Ultimately, if evidence is adequate, specific dietary intervention should be tested in disposed/susceptible study populations (e.g., disposed to allergic or autoimmune diseases or individuals at risk of malnourishment and stunting) to provide proof of applicability.

\section{Summary and Conclusions}

The infant gut microbiota undergoes significant temporal development during the first years of life. A multitude of factors such term of birth, mode of birth, antibiotics, contact with furred animals and siblings/family members, local environment, geographical location, and maternal and infant diet affect the trajectory of this development. Of these, infant/toddler diet has been identified as one of the most influential factors. The neonatal gut microbiota is initially characterized by random colonization of environmental microbes, but in a matter of days to weeks the gut ecosystem and the type milkfeeding selects for microbes adapted to the Gl environment and equipped to consume the indigestible (or incompletely digested) constituents of breast or formula milk. Breast milk feeding selects for specific $\mathrm{HMO}$-degrading Bifidobacterium species that vastly dominate the gut ecosystem and produce metabolites with beneficial effects likely contributing to the protection against Gl infections and supporting immune system development. On the contrary, formula-milk feeding is less selective and the gut microbiota of FF infants is more diverse with a higher frequency and proportion of protein degrading opportunistic pathogenic species. This may increase risk of $\mathrm{Gl}$ infections and the apparent mostly proteolytic metabolism is possibly detrimental to health as some of the metabolites are associated with kidney, liver, and cardiovascular diseases in adults.

As the infant transitions into complementary feeding the gut microbiota diversifies along with the increasing amounts of dietary fiber and proteins in the diet. Specifically, members of the bacterial families Lachnospiraceae, Ruminococcaceae, and Bacteroidaceae capable of consuming various dietary fibers from fruits, vegetables, breads and cereals, and possibly proteins from meat and dairy sources, increase in abundance during complementary feeding. This leads to a shift in gut metabolism with the appearance or increase of the SCFAs butyrate and propionate as well as the BCFAs. While the physiological relevance of these bacterial taxa and metabolites remains to be established in this early life context, recent research suggests that they may be important for healthy growth and development and protection against food allergies.

Clearly, the question of when to start introducing various complementary foods in order to achieve optimal health and gut microbiota progression is central. Early introduction, combined with reduced or lack of breastfeeding is presumably not beneficial, as it will disrupt the Bifidobacterium dominated gut community, associated with protection against infectious and immune-related diseases. On the other hand, very late introduction as observed in populations where food is scare and breastfeeding of the infant is prolonged without adequate complementary foods would also be expected to be adverse, given the inability of the gut microbiota to mature when deprived of key nutrients and the obvious negative implications for growth and development. Undoubtedly, further studies are required to improve our understanding of the intriguing links between complementary feeding, gut microbiota, and health.

\section{Conflict of Interest Statement}

The author declares no conflict of interest.

\section{Funding Sources}

The writing of the manuscript was supported by Nestlé Nutrition Institute.

\section{References}

1 Laursen MF, Bahl MI, Licht TR. Settlers of our inner surface: factors shaping the gut microbiota from birth to toddlerhood. FEMS Microbiol Rev. 2021;1:1-14.

2 Blaser MJ, Devkota S, McCoy KD, Relman DA, Yassour M, Young VB. Lessons learned from the prenatal microbiome controversy. Microbiome. $2021 \mathrm{Dec} ; 9(1): 8$

3 Bittinger K, Zhao C, Li Y, Ford E, Friedman ES, Ni J, et al. Bacterial colonization reprograms the neonatal gut metabolome. Nat Microbiol. 2020.

4 Pham VT, Lacroix C, Braegger CP, Chassard C. Early colonization of functional groups of microbes in the infant gut. Environ Microbiol. 2016 Jul;18(7):2246-58.
Laursen 
5 Obermajer T, Grabnar I, Benedik E, Tušar T, Robič Pikel T, Fidler Mis $\mathrm{N}$, et al. Microbes in infant gut development: placing abundance within environmental, clinical and growth parameters. Sci Rep. 2017 Dec;7(1):11230-14.

6 Jian C, Luukkonen P, Yki-Järvinen H, Salonen A, Korpela K. Quantitative PCR provides a simple and accessible method for quantitative microbiota profiling. PLoS One. 2020 Jan;15(1):e0227285.

7 Tsuji H, Matsuda K, Nomoto K. Counting the countless: bacterial quantification by targeting rRNA molecules to explore the human gut microbiota in health and disease. Front Microbiol. 2018 Jun; 9:1417.

8 Lozupone CA, Stombaugh JI, Gordon JI, Jansson JK, Knight R. Diversity, stability and resilience of the human gut microbiota. Nature. 2012 Sep;489(7415):220-30.

9 Rodríguez JM, Murphy K, Stanton C, Ross RP, Kober Ol, Juge N, et al. The composition of the gut microbiota throughout life, with an emphasis on early life. Microb Ecol Health Dis. 2015 Jan;26: 26050

10 Milani C, Duranti S, Bottacini F, Casey E, Turroni F, Mahony J, et al. The first microbial colonizers of the human gut: composition, activities, and health implications of the infant gut microbiota. Microbiol Mol Biol Rev. 2017 Dec; 81(4):e00036-17.

11 Stewart CJ, Ajami NJ, O'Brien JL, Hutchinson DS, Smith DP, Wong MC, et al. Temporal development of the gut microbiome in early childhood from the TEDDY study. Nature. 2018 Oct;562(7728): $583-8$.

12 Galazzo G, van Best N, Bervoets L, Dapaah IO, Savelkoul PH, Hornef $M W$, et al. Development of the microbiota and associations with birth mode, diet, and atopic disorders in a longitudinal analysis of stool samples, collected from infancy through early childhood. Gastroenterology. 2020 May;158(6):1584-96.

13 Laursen MF, Bahl MI, Michaelsen KF, Licht TR. First foods and gut microbes. Front Microbiol. 2017;8:356.

14 Victora CG, Bahl R, Barros AJ, França GV, Horton S, Krasevec J, et al. Breastfeeding in the 21st century: epidemiology, mechanisms, and lifelong effect. Lancet. 2016 Jan;387(10017):475-90.

15 Marchesi JR, Adams DH, Fava F, Hermes GD, Hirschfield GM, Hold $G$, et al. The gut microbiota and host health: a new clinical frontier. Gut. 2016 Feb;65(2):330-9.

16 Mosca A, Leclerc M, Hugot JP. Gut microbiota diversity and human diseases: Should we reintroduce key predators in our ecosystem? Front Microbiol. 2016 Mar; 7:455.

17 WHO. Breastfeeding [Internet]. [cited 2021 Mar 18]. Available from: https://www.who.int/health-topics/breastfeeding\#tab=tab_2.

18 Bode L. Human milk oligosaccharides: every baby needs a sugar mama. Glycobiology. 2012 Sep;22(9):1147-62.

19 Davis JC, Totten SM, Huang JO, Nagshbandi S, Kirmiz N, Garrido DA, et al. Identification of oligosaccharides in feces of breast-fed infants and their correlation with the gut microbial community. Mol Cell Proteomics. 2016;15(9):2987-3002.

20 Sakanaka M, Gotoh A, Yoshida K, Odamaki T, Koguchi H, Xiao JZ, et al. Varied pathways of infant gut-associated Bifidobacterium to assimilate human milk oligosaccharides: prevalence of the gene set and its correlation with bifidobacteria-rich microbiota formation. Nutrients. 2020 Dec;12(1):71.
21 Bunesova V, Lacroix C, Schwab C. Fucosyllactose and L-fucose utilization of infant Bifidobacterium longum and Bifidobacterium kashiwanohense. BMC Microbiol. 2016 Oct;16(1):248-12.

22 Özcan E, Sela DA. Inefficient metabolism of the human milk oligosaccharides lacto- $\mathrm{N}$-tetraose and lacto- $\mathrm{N}$-neotetraose shifts Bifidobacterium longum subsp. infantis physiology. Front Nutr. 2018 May; $5: 46$

23 Dedon LR, Özcan E, Rani A, Sela DA. Bifidobacterium infantis metabolizes 2'Fucosyllactose-derived and free fucose through a common catabolic pathway resulting in 1,2-propanediol secretion. Front Nutr. 2020 Nov; 7:583397.

24 Tsukuda N, Yahagi K, Hara T, Watanabe Y, Matsumoto H, Mori H, et al. Key bacterial taxa and metabolic pathways affecting gut short-chain fatty acid profiles in early life. ISME J. 2021 Mar:1-17.

25 Pichler MJ, Yamada C, Shuoker B, Alvarez-Silva C, Gotoh A, Leth $\mathrm{ML}$, et al. Butyrate producing colonic Clostridiales metabolise human milk oligosaccharides and cross feed on mucin via conserved pathways. Nat Commun. 2020;11(1):3285.

26 Marcobal A, Barboza M, Sonnenburg ED, Pudlo N, Martens EC, Desai $P$, et al. Bacteroides in the infant gut consume milk oligosaccharides via mucus-utilization pathways. Cell Host Microbe. 2011 Nov;10(5):507-14

27 Rodríguez-Díaz J, Monedero V, Yebra MJ. Utilization of natural fucosylated oligosaccharides by three novel $a$-L-fucosidases from a probiotic lactobacillus casei strain. Appl Environ Microbiol. 2011 Jan;77(2):703-5.

28 Turroni F, Peano C, Pass DA, Foroni E, Severgnini M, Claesson MJ, et al. Diversity of bifidobacteria within the infant gut microbiota. PLoS One. 2012 Jan;7(5):e36957.

29 Laursen MF, Sakanaka M, von Burg N, Andersen D, Mörbe U, Rivollier A, et al. Breastmilk-promoted bifidobacteria produce aromatic lactic acids in the infant gut. bioRxiv. 2020 Jan.

30 Matsuki T, Yahagi K, Mori H, Matsumoto H, Hara T, Tajima S, et al. A key genetic factor for fucosyllactose utilization affects infant gut microbiota development. Nat Commun. 2016 Sep;7(1):11939.

31 Frese SA, Hutton AA, Contreras LN, Shaw CA, Palumbo MC, Casaburi $G$, et al. Persistence of supplemented Bifidobacterium longum subsp. infantis EVC001 in breastfed infants. mSphere. 2017 Dec;2(6):e00501-17

32 Henrick BM, Hutton AA, Palumbo MC, Casaburi G, Mitchell RD, Underwood MA, et al. Elevated fecal $\mathrm{pH}$ indicates a profound change in the breastfed infant gut microbiome due to reduction of Bifidobacterium over the past century. mSphere. 2018 Mar; 3(2): e00041-18.

33 Fukuda S, Toh H, Hase K, Oshima K, Nakanishi Y, Yoshimura K, et al. Bifidobacteria can protect from enteropathogenic infection through production of acetate. Nature. 2011;469(7331):543-7.

34 Peters A, Krumbholz P, Jäger E, Heintz-Buschart A, Cakir MV, Rothemund $\mathrm{S}$, et al. Metabolites of lactic acid bacteria present in fermented foods are highly potent agonists of human hydroxycarboxylic acid receptor 3. PLoS Genet. 2019 May;15(5):e1008145.

35 Ehrlich AM, Pacheco AR, Henrick BM, Taft D, Xu G, Huda MN, et al. Indole-3-lactic acid associated with Bifidobacterium-dominated microbiota significantly decreases inflammation in intestinal epithelial cells. BMC Microbiol. 2020 Dec;20(1):357. 
36 Meng D, Sommella E, Salviati E, Campiglia P, Ganguli K, Djebali K, et al. Indole-3-lactic acid, a metabolite of tryptophan, secreted by Bifidobacterium longum subspecies infantis is anti-inflammatory in the immature intestine. Pediatr Res. 2020 Aug;88(2):209-17.

37 Henrick BM, Chew S, Casaburi G, Brown HK, Frese SA, Zhou Y, et al. Colonization by B. infantis EVC001 modulates enteric inflammation in exclusively BF infants. Pediatr Res. 2019 Dec;86(6):74957.

38 Casaburi G, Duar RM, Brown H, Mitchell RD, Kazi S, Chew S, et al. Metagenomic insights of the infant microbiome community structure and function across multiple sites in the United States. Sci Rep. 2021 Dec;11(1):1472.

39 Forsgren M, Isolauri E, Salminen S, Rautava S. Late preterm birth has direct and indirect effects on infant gut microbiota development during the first six months of life. Acta Paediatr. 2017 Jul; 106(7):1103-9.

40 Henrick BM, Rodriguez L, Lakshmikanth T, Pou C, Henckel E, Olin A, et al. Bifidobacteria-mediated immune system imprinting early in life. bioRxiv. 2020 Oct.

41 Stokholm J, Blaser MJ, Thorsen J, Rasmussen MA, Waage J, Vinding RK, et al. Maturation of the gut microbiome and risk of asthma in childhood. Nat Commun. 2018 Dec;9(1):141.

42 Vatanen T, Kostic AD, d'Hennezel E, Siljander H, Franzosa EA, Yassour $M$, et al. Variation in microbiome LPS immunogenicity contributes to autoimmunity in humans. Cell. 2016 May;165(4):84253.

43 Alcon-Giner C, Dalby MJ, Caim S, Ketskemety J, Shaw A, Sim K, et al. Microbiota supplementation with bifidobacterium and lactobacillus modifies the preterm infant gut microbiota and metabolome: an Observational Study. Cell Rep Med. 2020 Aug;1(5): 100077.

44 Nguyen M, Holdbrooks H, Mishra P, Abrantes MA, Eskew S, Garma $M$, et al. Impact of probiotic B. infantis EVC001 feeding in premature infants on the gut microbiome, nosocomially acquired antibiotic resistance, and enteric inflammation. Front Pediatr. 2021 Feb; 9:618009.

45 Penders J, Thijs C, van den Brandt PA, Kummeling I, Snijders B, Stelma F, et al. Gut microbiota composition and development of atopic manifestations in infancy: the KOALA Birth Cohort Study. Gut. 2007 May; 56(5):661-7.

46 Bäckhed F, Roswall J, Peng Y, Feng Q, Jia H, Kovatcheva-Datchary $P$, et al. Dynamics and stabilization of the human gut microbiome during the first year of life. Cell Host Microbe. 2015 May;17(5): $690-703$.

47 Benno Y, Sawada K, Mitsuoka T. The intestinal microflora of infants: composition of fecal flora in breast-fed and bottle-fed infants. Microbiol Immunol. 1984 Sep;28(9):975-86.

48 Rossi M, Corradini C, Amaretti A, Nicolini M, Pompei A, Zanoni S, et al. Fermentation of fructooligosaccharides and inulin by bifidobacteria: a comparative study of pure and fecal cultures. Appl Environ Microbiol. 2005 Oct;71(10):6150-8.

49 Akiyama T, Kimura K, Hatano H. Diverse galactooligosaccharides consumption by bifidobacteria: Implications of $\beta$-galactosidaseLacS operon. Biosci Biotechnol Biochem. 2015 Apr;79(4):664-72.
50 Schwab C, Gänzle M. Lactic acid bacteria fermentation of human milk oligosaccharide components, human milk oligosaccharides and galactooligosaccharides. FEMS Microbiol Lett. 2011 Feb; 315(2):141-8.

51 Ma C, Wasti S, Huang S, Zhang Z, Mishra R, Jiang S, et al. The gut microbiome stability is altered by probiotic ingestion and improved by the continuous supplementation of galactooligosaccharide. Gut Microbes. 2020 Nov;12(1):1-13.

52 Chow J, Panasevich MR, Alexander D, Vester Boler BM, Rossoni Serao MC, Faber TA, et al. Fecal metabolomics of healthy breastfed versus formula-fed infants before and during in vitro batch culture fermentation. J Proteome Res. 2014 May;13(5):2534-42.

53 He X, Parenti M, Grip T, Lönnerdal B, Timby N, Domellöf M, et al. Fecal microbiome and metabolome of infants fed bovine MFGM supplemented formula or standard formula with breast-fed infants as reference: a randomized controlled trial. Sci Rep. 2019 Dec;9(1):1-14.

54 Gryp T, Vanholder R, Vaneechoutte M, Glorieux G. p-Cresyl Sulfate. Toxins. 2017 Jan; 9(2):52.

55 Nemet I, Saha PP, Gupta N, Zhu W, Romano KA, Skye SM, et al. A cardiovascular disease-linked gut microbial metabolite acts via adrenergic receptors. Cell. 2020 Mar;180(5):862-e22.

56 Delzenne NM, Bindels LB. Microbiome metabolomics reveals new drivers of human liver steatosis. Nat Med. 2018 Jul;24(7):906-7.

57 Saraf MK, Piccolo BD, Bowlin AK, Mercer KE, LeRoith T, Chintapalli SV, et al. Formula diet driven microbiota shifts tryptophan metabolism from serotonin to tryptamine in neonatal porcine colon. Microbiome. 2017 Jul;5(1):77.

58 Fernández-Reina A, Urdiales J, Sánchez-Jiménez F. What we know and what we need to know about aromatic and cationic biogenic amines in the gastrointestinal tract. Foods. 2018 Sep;7(9): 145.

59 Daniel Collins J, Noerrung B, Budka H, Andreoletti O, Buncic S, Griffin J, et al. Scientific Opinion on risk based control of biogenic amine formation in fermented foods. EFSA J. 2011 Oct;9(10): 2393.

60 Shaw AG, Cornwell E, Sim K, Thrower H, Scott H, Brown JCS, et al. Dynamics of toxigenic Clostridium perfringens colonisation in a cohort of prematurely born neonatal infants. BMC Pediatr. 2020 Feb;20(1):75.

61 Kuiper GA, van Prehn J, Ang W, Kneepkens F, van der Schoor S, de Meij T. Clostridium difficile infections in young infants: case presentations and literature review. IDCases. 2017 Jan;10:7-11.

62 Rahman SF, Olm MR, Morowitz MJ, Banfield JF. Machine learning leveraging genomes from metagenomes identifies influential antibiotic resistance genes in the infant gut microbiome. mSystems. 2018 Jan;3(1):e00123-17.

63 Pärnänen K, Hultman J, Satokari R, Rautava S, Lamendella R, Wright $\mathrm{J}$, et al. Formula alters preterm infant gut microbiota and increases its antibiotic resistance load. bioRxiv. 2019 Sep:782441.

64 Ossa JC, Yáñez D, Valenzuela R, Gallardo P, Lucero Y, Farfán MJ. Intestinal inflammation in Chilean infants fed with bovine formula vs. breast milk and its association with their gut microbiota. Front Cell Infect Microbiol. 2018 Jun; 8:190. 
65 Salminen S, Stahl B, Vinderola G, Szajewska H. Infant formula supplemented with biotics: current knowledge and future perspectives. Nutrients. 2020 Jul;12(7):1-20.

66 Berger B, Porta N, Foata F, Grathwohl D, Delley M, Moine D, et al. Linking human milk oligosaccharides, infant fecal community types, and later risk to require antibiotics. MBio. 2020 Apr;11(2):17.

67 WHO. Complementary feeding: global [Internet]. 2020 [cited 2021 Mar 16]. Available from: https://www.who.int/health-topics/complementary-feeding\#tab=tab_2.

68 Castenmiller J, De Henauw S, Hirsch-Ernst K-I, Kearney J, Maciuk A, Mangelsdorf I, et al. Scientific Opinion appropriate age range for introduction of complementary feeding into an infant's diet EFSA panel on nutrition, novel foods and food allergens (NDA). 2019.

69 Schiess S, Grote V, Scaglioni S, Luque V, Martin F, Stolarczyk A, et al. Introduction of complementary feeding in 5 european countries. J Pediatr Gastroenterol Nutr. 2010 Jan;50(1):92-8.

70 Przyrembel $\mathrm{H}$. Timing of introduction of complementary food: Short- and long-term health consequences. Ann Nutr Metab. 2012;60(Suppl 2):8-20.

71 Krawiec M, Fisher HR, Du Toit G, Bahnson HT, Lack G. Overview of oral tolerance induction for prevention of food allergy - Where are we now? Allergy. 2021 Mar.

72 Gehrig JL, Venkatesh S, Chang HW, Hibberd MC, Kung VL, Cheng $\mathrm{J}$, et al. Effects of microbiota-directed foods in gnotobiotic animals and undernourished children. Science. 2019 Jul(6449):365.

73 Blanton LV, Charbonneau MR, Salih T, Barratt MJ, Venkatesh S, Ilkaveya $\mathrm{O}$, et al. Gut bacteria that prevent growth impairments transmitted by microbiota from malnourished children. Science. 2016;351(6275):aad3311.

74 Feehley T, Plunkett CH, Bao R, Choi Hong SM, Culleen E, BeldaFerre $P$, et al. Healthy infants harbor intestinal bacteria that protect against food allergy. Nat Med. 2019 Mar;25(3):448-53.

75 Hong SW, O E, Lee JY, Lee M, Han D, Ko HJ, et al. Food antigens drive spontaneous IgE elevation in the absence of commensal microbiota. Sci Adv. 2019 May;5(5):eaaw1507.

76 Cummings $\mathrm{JH}$, Macfarlane GT. The control and consequences of bacterial fermentation in the human colon. J Appl Bacteriol. 1991 Jun;70(6):443-59.

77 Scott KP, Duncan SH, Flint HJ. Dietary fibre and the gut microbiota. Nutr Bull. 2008;33(3):201-11.

78 Appert O, Garcia AR, Frei R, Roduit C, Constancias F, Neuzil-Bunesova $\mathrm{V}$, et al. Initial butyrate producers during infant gut microbiota development are endospore formers. Environ Microbiol. 2020 Sep;22(9):3909-21.

79 Louis $\mathrm{P}$, Flint HJ. Formation of propionate and butyrate by the human colonic microbiota. Environ Microbiol. 2017 Jan;19(1):29-41.

80 Flint HJ, Scott KP, Duncan SH, Louis P, Forano E. Microbial degradation of complex carbohydrates in the gut. Gut Microbes. 2012 Jul-Aug;3(4):289-306.

81 Smith EA, Macfarlane GT. Enumeration of amino acid fermenting bacteria in the human large intestine: effects of $\mathrm{pH}$ and starch on peptide metabolism and dissimilation of amino acids. FEMS Microbiol Ecol. 1998 Apr;25(4):355-68.
82 Koh A, De Vadder F, Kovatcheva-Datchary P, Bäckhed F. From dietary fiber to host physiology: short-chain fatty acids as key bacterial metabolites. Cell. 2016 Jun;165(6):1332-45.

83 Rios-Covian D, González S, Nogacka AM, Arboleya S, Salazar N, Gueimonde M, et al. An overview on fecal branched short-chain fatty acids along human life and as related with body mass index: associated dietary and anthropometric factors. Front Microbiol. 2020 May;11:973.

84 Roger LC, McCartney AL. Longitudinal investigation of the faecal microbiota of healthy full-term infants using fluorescence in situ hybridization and denaturing gradient gel electrophoresis. Microbiology. 2010;156(Pt 11):3317-28.

85 Thompson AL, Monteagudo-Mera A, Cadenas MB, Lampl ML, Azcarate-Peril MA. Milk- and solid-feeding practices and daycare attendance are associated with differences in bacterial diversity, predominant communities, and metabolic and immune function of the infant gut microbiome. Front Cell Infect Microbiol. 2015;5: 3.

86 Differding MK, Benjamin-Neelon SE, Hoyo C, Østbye T, Mueller NT. Timing of complementary feeding is associated with gut microbiota diversity and composition and short chain fatty acid concentrations over the first year of life. BMC Microbiol. 2020;20(1): $56-13$.

87 Leong C, Haszard JJ, Lawley B, Otal A, Taylor RW, Szymlek-Gay EA, et al. Mediation analysis as a means of identifying dietary components that differentially affect the fecal microbiota of infants weaned by modified baby-led and traditional approaches. Appl Environ Microbiol. 2018 Sep; 84(18):e00914-18.

88 Andersen LB, Pipper CB, Trolle E, Bro R, Larnkjær A, Carlsen EM, et al. Maternal obesity and offspring dietary patterns at 9 months of age. Eur J Clin Nutr. 2015 Jun;69(6):668-75.

89 Laursen MF, Andersen LB, Michaelsen KF, Mølgaard C, Trolle E, Bahl MI, et al. Infant gut microbiota development is driven by transition to family foods independent of maternal obesity. mSphere. 2016 Feb;1(1):e00069-15.

90 Tang M, Frank D, Hendricks A, Ir D, Krebs N. Protein intake during early complementary feeding affects the gut microbiota in U.S. formula-fed infants (FSO4-03-19). Curr Dev Nutr. 2019;3(Suppl 1): 930.

91 Krebs NF, Sherlock LG, Westcott J, Culbertson D, Hambidge KM, Feazel $L M$, et al. Effects of different complementary feeding regimens on iron status and enteric microbiota in BF infants. J Pediatr. 2013;163(2):416-23.

92 Ordiz MI, Janssen S, Humphrey G, Ackermann G, Stephenson K, Agapova S, et al. The effect of legume supplementation on the gut microbiota in rural Malawian infants aged 6 to 12 months. Am J Clin Nutr. 2020;111(4):884-92.

93 Qasem W, Azad MB, Hossain Z, Azad E, Jorgensen S, Castillo San Juan $\mathrm{S}$, et al. Assessment of complementary feeding of Canadian infants: effects on microbiome \& oxidative stress, a randomized controlled trial. BMC Pediatr. 2017;17(1):54.

94 Subramanian S, Huq S, Yatsunenko T, Haque R, Mahfuz M, Alam MA, et al. Persistent gut microbiota immaturity in malnourished Bangladeshi children. Nature. 2014;510(7505):417-21. 
95 Raman AS, Gehrig JL, Venkatesh S, Chang HW, Hibberd MC, Subramanian $\mathrm{S}$, et al. A sparse covarying unit that describes healthy and impaired human gut microbiota development. Science. 2019; 365(6449):eaau4735.

96 Planer JD, Peng Y, Kau AL, Blanton LV, Ndao IM, Tarr PI, et al. Development of the gut microbiota and mucosal IgA responses in twins and gnotobiotic mice. Nature. 2016 May;534(7606):263-6.
97 Yatsunenko T, Rey FE, Manary MJ, Trehan I, Dominguez-Bello MG, Contreras $\mathrm{M}$, et al. Human gut microbiome viewed across age and geography. Nature. 2012 Jun;486(7402):222-7. 